\title{
Competencia intercultural en la enseñanza de lenguas extranjeras
}

\author{
$\mathrm{M}^{\mathrm{a}}$ Silvina Paricio TATO \\ IES plurilingüe Elviña (A Coruña)
}

Recibido: 11 octubre 2012 / Aceptado: 28 febrero de 2013

ISSN: $1697-7467$

\begin{abstract}
RESUMEN: El presente artículo pretende clarificar qué se entiende por competencia intercultural en la enseñanza de lenguas extranjeras. En un primer apartado introductorio se hace referencia a diversos trabajos que se han ocupado de investigar las relaciones entre lengua y cultura y a las razones que han conducido a la necesidad de potenciar una enseñanza integrada de ambas. Posteriormente, se apuntan algunos conceptos clave sobre el enfoque o dimensión intercultural aplicada a la enseñanza de lenguas y se proporciona un marco conceptual en el que el que se apoya dicha dimensión. El artículo concluye con una breve indicación sobre el papel que compete al profesorado.

Palabras clave: enseñanza de lenguas extranjeras, competencia intercultural, currículum, formación del profesorado.
\end{abstract}

\section{Intercultural Competence in Foreign Language Teaching}

\begin{abstract}
The present article seeks to clarify what is meant by intercultural competence when teaching a foreign language. An introductory paragraph refers to the various researches that have focused on studying the relationships between language and culture, and the reasons that have led to the need to promote integrated teaching of both. Next, it points out some key concepts on the approach or intercultural dimension applied to language teaching and provides a conceptual framework that supports this dimension. The article concludes with a brief indication concerning the teachers'role.
\end{abstract}

Keywords: foreign language teaching, intercultural competence, curriculum, teacher training/development.

\section{InTRODUCCIÓN}

Desde hace un par de décadas, no es infrecuente encontrar en los estudios teóricos sobre la enseñanza de lenguas extranjeras términos como los de "componente cultural", "dimensión intercultural", "competencia intercultural", "competencia comunicativa intercultural" y también continuas referencias al importante papel que corresponde a la cultura en el proceso de enseñanza-aprendizaje de una lengua. Podemos mencionar, entre otros, los trabajos de Buttjes y Byram (1991), Byram (1997), Byram, Morgan y otros (1994), Byram y Risager (1999), Byram y Fleming (2001), Kramsch (1993, 1998 y 2001), Sercu (2005), o Zárate (1986 y 1995). A ellos hemos de añadir el importante papel desempeñado por el Consejo de Europa en su división dedicada al estudio y promoción de la enseñanza de lenguas, una de cuyas publicaciones, el Marco de referencia europeo para el aprendizaje, la enseñanza 
y la evaluación de las lenguas (Instituto Cervantes, 2002), se ha convertido en una especie de "faro" por el que ha de guiarse la enseñanza-aprendizaje de lenguas en Europa, ya sea como lenguas extranjeras o como segundas lenguas.

De los estudios mencionados cabe destacar que todos ellos abordan la cuestión de la relación entre lengua y cultura, proporcionando orientaciones y exposiciones acerca de la necesidad de abordar la enseñanza de ambas de un modo integral. La evolución de una enseñanza que ponía el énfasis en la competencia comunicativa hacia una orientación intercultural obedecería al nuevo contexto social en el que estamos inmersos. Por una parte, están la internacionalización de los mercados y la globalización cultural, que han incrementado considerablemente la necesidad de establecer intercambios con gentes de distintos países; por otra, y como consecuencia de los fenómenos migratorios, nos encontramos, dentro del contexto de cada país, con una importante diversidad cultural, con gentes pertenecientes a distintas latitudes con las que entramos en contacto en nuestra vida diaria. Nuestras sociedades se han vuelto cada vez más multiculturales. Así, por ejemplo, la constitución de la Unión Europea ha dado lugar a una circulación por los distintos países que la conforman de numerosas personas que desean encontrar trabajo en un país distinto del propio. También se han incrementado de manera notable los viajes turísticos o por razón de estudios. Estos hechos explican que en el seno de la Unión se promueva el conocimiento de las lenguas comunitarias y que ésta se fije como meta que los ciudadanos y ciudadanas europeos aprendan dos, a mayores de la propia. Queda claro, pues, cuál es el motivo por el que aprender lenguas se vea como una necesidad que conlleva nuevos planteamientos metodológicos y didácticos en ese terreno. Esta institución considera la diversidad lingüística europea como un rico patrimonio que hay que preservar.

Sercu (2005) apunta acertadamente que la incorporación de objetivos interculturales en los currículos no es algo exclusivo de la enseñanza de lenguas, sino que afecta a una gran diversidad de materias, y que la formación en lenguas extranjeras es, por definición, intercultural. Llevar una lengua extranjera al aula significa poner en contacto al alumnado con un mundo culturalmente diferente al propio. Se requiere, por tanto, que el profesorado de lenguas explote este potencial y promueva la adquisición de una competencia comunicativa intercultural. El objetivo del aprendizaje en este campo no se define ya en términos de adquisición de una competencia comunicativa.

Como señalan Byram, Nichols y Stevens (2001) no es difícil persuadir al profesorado de lenguas de que es importante enseñar lenguas y culturas como un todo integrado. En realidad, la lengua es el vehículo de expresión de una cultura diferente a la propia, algo que, en nuestra opinión, todo docente percibe sin dificultad porque lo tiene, por así decirlo, interiorizado. Sin embargo, podemos afirmar que hasta hace no muchos años -e incluso hasta hoy- esta enseñanza integrada de lengua y cultura no se ha visto reflejada en la práctica docente. En realidad, la forma de enfocar el aprendizaje de la(s) cultura(s) asociada(s) a una determinada lengua ha ido variando a lo largo del tiempo. Durante un buen número de años, la cultura se identificaba con la Cultura con C mayúscula, esto es, las grandes realizaciones de un determinado pueblo en el campo de la historia, las artes, la literatura, etc. Hoy en día, el concepto de cultura que prevalece es el antropológico, esto es, el de cultura con c minúscula, entendiendo por tal las costumbres, tradiciones y modos de vida cotidiana de una comunidad, etc. 
Ocurre que la dimensión cultural, entendida en el sentido de Cultura y denominada como "background studies" en inglés, "Civilisation" en francés y "Landeskunde" en alemán, ha formado parte tradicionalmente del pensamiento del profesor, aunque, en épocas recientes, no ha sido parte de su práctica. A partir de los años 70, con el auge de los enfoques comunicativos, método imperante en la enseñanza de idiomas, ésta se limitaba a la dimensión puramente lingüística, a cifrar su objetivo en adquirir una competencia comunicativa, obviándose así la dimensión cultural, reducida a un papel meramente anecdótico, con la presencia de pequeños "flashes" de informaciones culturales sobre el país o países donde se habla la lengua en cuestión en los materiales didácticos más implantados en el aula, los libros de texto o métodos. Byram, Nichols y Stevens (2001) apuntan que ese no prestar atención a la cultura sería un reflejo de la teoría por la que se guiaba la enseñanza de las lenguas en épocas recientes. Así, la enseñanza de las lenguas se habría visto influida por la lingüística pura y aplicada $\mathrm{y}$, además, el impacto de los distintos métodos vigentes en cada momento socio-histórico habría sido importante.

En base a la anterior afirmación, puede decirse que las teorías se han centrado en desarrollar nuevos modos de presentar la lengua, desarrollar habilidades lingüísticas, crear fluidez lingüística y exactitud, y enseñar al alumnado cómo emplear la lengua de modo adecuado de acuerdo con el análisis de las prácticas sociolingüísticas. Una breve síntesis del papel ocupado por el componente sociocultural en los diversos métodos de enseñanza de las lenguas extranjeras a lo largo del pasado siglo puede consultarse en Neuner (2003: 19-23) y Rico (2005:88-92). Neuner (2003) señala que estas variaciones en el tratamiento del enfoque intercultural obedecerían a los cambios acontecidos en el contexto sociopolítico.

Hoy en día, muchos currículos y programas se refieren a la importancia del aprendizaje intercultural y, más recientemente, a la competencia intercultural. Pese a ello, existe todavía una carencia de "buenas prácticas" y una atención insuficiente a la dimensión cultural/intercultural en la formación del profesorado. Nuestra intención, en el presente artículo, es clarificar el concepto de competencia intercultural/competencia comunicativa intercultural, y efectuar algunas indicaciones destinadas al profesorado sobre cómo desarrollar ésta en el aula.

\section{EL ENFOQUE INTERCULTURAL EN LA ENSEÑANZA DE LENGUAS: CONCEPTOS CLAVE}

Como hemos apuntado en el párrafo anterior, la mayoría de los currículos de lenguas de los países europeos incluyen, de alguna manera, otras funciones a desempeñar por éstas en el momento actual a mayores de la simple adquisición de una competencia lingüística. Así lo confirmaba, hace ya algunos años, un estudio sobre la enseñanza de lenguas en el contexto escolar europeo (Eurydice, 2001) difundido el mismo año en que se publicó el Marco europeo de referencia (Instituto Cervantes, 2002 ${ }^{1}$ ). De acuerdo con la mencionada investigación, la práctica totalidad de los programas escolares europeos contemplaba ya entonces en sus formulaciones el estrecho vínculo existente entre el aprendizaje de una lengua extranjera y la apertura hacia la(s) cultura(s) de la comunidad o comunidades donde se habla. Mediante

\footnotetext{
${ }^{1}$ El original en inglés se publicó en el año 2001.
} 
esta apertura no sólo se posibilitaría la adquisición de nuevos conocimientos culturales, sino que se facilitaría también el desarrollo de actitudes de respeto, tolerancia y comprensión del "otro" en su diferencia. Por regla general, los programas vigentes en el momento en que se llevó a cabo la investigación comenzaban por una invitación a que el alumnado tomase conciencia de las relaciones existentes entre lengua y cultura. Ahora bien, existía una gran variedad, según los países, a la hora de precisar los conocimientos culturales a adquirir por el alumnado, pudiendo referirse éstos tanto a los usos y costumbres de la vida cotidiana como a la geografía y a las instituciones políticas del país o países donde se hablaba la lengua objeto de estudio. En algún programa se aludía al desarrollo del espíritu crítico y reflexivo en el alumnado, aspecto éste que podía verse favorecido mediante el estudio de los elementos culturales de otros países. Se pensaba así que dicha reflexión podría conducir a los alumnos y alumnas al desarrollo de una visión más objetiva y crítica sobre la propia cultura. Así pues, junto con los intereses comunicativos y reflexivos, los currículos subrayaban cómo a través del estudio de las distintas facetas de una cultura se favorecía el desarrollo de actitudes de respeto y comprensión hacia la alteridad, siendo éste un objetivo general a lograr que podía presentar distintos matices:

Es posible inscribir todos los matices de este objetivo general en el interior de un continuo enmarcado, por un lado, por la idea de curiosidad y de apertura de espíritu, y, por otro, por la de empatía por el otro. Entre estos dos extremos, se sitúan invitaciones a una mayor tolerancia, a una mayor apertura hacia los valores de los otros, a la superación de los estereotipos, a la aceptación del otro en sus diferencias. La adquisición de valores como la solidaridad o incluso la adopción de actitudes positivas con respecto a los valores europeos están presentes igualmente en algunos programas. (Eurydice, 2001: 167).

Tras este breve recorrido por los programas europeos, nos centraremos ahora en el currículo de Lengua Extranjera para la Educación Secundaria Obligatoria vigente en nuestro país (Real Decreto 1631/2006: 741-750) ${ }^{2}$. En él se reconoce que el eje de esta enseñanza tiene como objetivo el dominio de las clásicas habilidades lingüísticas productivas: hablar, conversar y escribir; y las receptivas: escuchar y comprender, leer y comprender. Sin embargo, se apunta claramente que el aprendizaje de una lengua extranjera trasciende el marco lingüístico e implica un valor formativo integral para el alumnado, en tanto que favorece el respeto, el interés y la comunicación con hablantes de otras culturas y desarrolla la consciencia intercultural. Se pone además el énfasis en su componente actitudinal, puesto que contribuye al desarrollo de actitudes positivas y receptivas hacia otras culturas y, a la vez, ayuda a comprender y valorar la lengua o lenguas propias. El currículo se estructura así en cuatro bloques: 1) Escuchar, hablar y conversar; 2) Leer y escribir; 3) Conocimiento de la lengua; y 4) Aspectos socioculturales y consciencia intercultural. Es este último bloque de contenidos el que apunta más directamente a la competencia intercultural, dado que contribuye "a que el alumnado conozca costumbres, formas de relación social, rasgos y particularidades de los países en los que se habla la lengua extranjera, en definitiva, formas de vida diferentes

\footnotetext{
${ }^{2}$ Un estudio más completo de la dimensión intercultural en este texto legal puede consultarse en Paricio
} (2011). 
a las suyas" (Ibídem: 742). Se entiende que dicho conocimiento fomentará la tolerancia y la aceptación, acrecentando su interés por distintas realidades sociales y culturales y, con ello, "facilitará la comunicación intercultural porque las lenguas son portadoras de contenidos, rasgos y marcas de las culturas de las cuales son el medio de expresión" (Ibídem)3.

Según apunta Areizaga (2001:162), hoy en día existe un consenso acerca del papel desempeñado por las lenguas extranjeras, a las que se atribuyen unos objetivos educativos de carácter formativo que implican ir más allá de la adquisición de una competencia lingüística y cuya meta es "formar ciudadanos para una sociedad multicultural y multilingüe... El concepto de competencia comunicativa intercultural intenta responder a la necesidad de ofrecer un modelo para la integración de lengua y cultura en la enseñanza de lenguas".

Para abordar el concepto de competencia comunicativa intercultural, nos apoyaremos, por una parte, en el Marco europeo de referencia para el aprendizaje, la enseñanza y la evaluación de lenguas (Instituto Cervantes, 2002), donde nos referiremos a la nueva concepción del hecho comunicativo que subyace a sus planteamientos; y, por otra, en la línea iniciada y desarrollada en el contexto europeo por Byram y sus numerosos colaboradores en lo que respecta a la competencia intercultural.

Según hemos indicado anteriormente, los enfoques comunicativos, predominantes hasta no hace mucho en la enseñanza de las lenguas, han sido objeto de críticas por privilegiar una concepción instrumental del aprendizaje de éstas, obviando o reduciendo a un papel anecdótico la dimensión cultural. De acuerdo con Isisag (2010), el enfoque comunicativo fallaba sobre todo en dos aspectos: 1) no reconociendo plenamente los lazos entre lengua y cultura; y 2) no siendo consciente de que el aprendizaje de otra cultura era insuficiente para promover la comprensión entre culturas, ya que para alcanzar esta última finalidad, se requiere tener una consciencia de la propia cultura.

A estos factores hemos de añadir que el modelo a seguir era el del 'hablante nativo', esto es, se planteaba que el alumnado adquiriese un dominio de la lengua similar al de éste. Desde la óptica intercultural, la meta principal es "convertir a las personas que aprenden una lengua en hablantes o mediadores interculturales capaces de introducirse en un marco complejo y en un contexto de identidades múltiples, así como de evitar los estereotipos que acompañan generalmente la percepción del otro al asignarle una única identidad" (Instituto Cervantes: 12, cit. en Paricio, 2004: 4). La apuesta por el "hablante intercultural" ha sido defendida por diversos especialistas en distintas publicaciones (Byram, 1997: 31; Byram y Risager, 1999: 4; Byram, Gribkova y Starkey (2002: 9-10) y, especialmente, Kramsch, 2001: 23-36).

Otra característica importante de la dimensión intercultural es que la cultura no se concibe como algo reificado y homogéneo, sino como algo dinámico, en continuo cambio y caracterizado por la propia diversidad interna. La misión del profesorado no es ya transmitir un corpus de conocimientos más o menos cerrado sobre la(s) cultura(s) del país o países donde se habla la lengua objeto de estudio, sino que ha de fomentar el aprendizaje autónomo del alumnado. El papel del docente es el de mediador entre el alumnado y la cultura extranjera. Como indica Sercu (2002), este enfoque considera que, junto con la adquisición

\footnotetext{
${ }^{3}$ Uno de los autores que más se ha ocupado de relacionar la enseñanza y el aprendizaje de lenguas con la educación para la ciudadanía es Starkey $(2003,2005)$.
} 
de una competencia lingüística comunicativa, el profesorado ha de tener en cuenta como objetivo pedagógico promover en el alumnado un interés en, un conocimiento sobre y una apertura hacia las culturas, gentes y países extranjeros:

Presentar al alumnado múltiples perspectivas [la variedad interna existente dentro de la propia cultura y de la extranjera] promoverá una visión dinámica de las culturas, y le ayudará a comprender que todas las culturas se ven continuamente influidas por otras culturas y no pueden ser consideradas de un modo "territorializado", como estando unidas a una parte geográfica particular del mundo o como encerradas entre los límites de un estado-nación. (Sercu, 2002: 69)

Byram, Nichols y Stevens (2001), por su parte, insisten en que el trabajo del profesorado no es proporcionar una información exhaustiva e intentar introducir la sociedad extranjera en la clase para que el alumnado aprenda vicariamente. Su verdadera tarea es facilitar la interacción del alumnado con algunos aspectos de otra sociedad y sus culturas con el objetivo de relativizar la comprensión de los propios valores culturales, creencias y comportamientos, y animarlo a investigar la alteridad que le rodea, ya sea en su entorno más inmediato, ya sea en el contexto del compromiso con la alteridad que la internacionalización y la globalización han traído a su mundo.

Abdallah-Pretceille (1998: 50) indica que se ha pasado de una enseñanza de la civilización, concebida como un saber normativo y descriptivo, a un aprendizaje de un saber-hacer de tipo comprensivo e interpretativo. Esto supone, en el plano pedagógico, el final de las técnicas expositivas y magistrales en favor del recurso a métodos activos que permitan aprender a estudiar documentos auténticos y analizar distintas situaciones de comunicación. La simple adición de hechos culturales tomados de manera aislada es tan ineficaz, para acercarse a una cultura, como la memorización de palabras fuera de contexto para aprender una lengua.

Risager (2001: 244) señala la década de los ochenta como el momento en que la enseñanza de lenguas empieza a verse influida por la perspectiva intercultural. Esta última se fundamenta en un concepto de cultura que acepta que las diferentes culturas están relacionadas estructuralmente entre sí. Se contempla el encuentro o interacción entre culturas, "y sus intentos por conocerse, entenderse y reconocerse". Según esta misma autora, el enfoque intercultural tiene en cuenta tanto la cultura del país o países objeto de estudio como la del país del estudiante. Incluye asimismo la comparación de los países objeto de estudio y el del alumnado, invitando a este último a adoptar una actitud crítica con respecto a ambas culturas. El propósito último consiste en desarrollar una competencia intercultural y comunicativa que permita al estudiante actuar como mediador entre las dos culturas.

\section{Marco conceptual de la COMPetencia intercultural}

Analizaremos ahora el marco teórico en que se asienta la competencia intercultural a través del estudio del Marco europeo de referencia (Instituto Cervantes, 2002) y de los textos de diversos especialistas que se han ocupado también de esta temática. 
El Marco europeo se hace eco de una nueva concepción del hecho comunicativo que pone su énfasis en el papel de los individuos como actores sociales dotados de múltiples identidades (nacional, local, regional, profesional, etc.) y de un bagaje de conocimientos sobre el mundo y aptitudes y destrezas que entran en juego en la comunicación.

La comunicación apela al ser humano en su totalidad ... Como agente social, cada individuo establece relaciones con un amplio conjunto de grupos sociales superpuestos que unidos definen la identidad. En un enfoque intercultural, uno de los objetivos fundamentales de la educación en la lengua es el impulso del desarrollo favorable de la personalidad del alumno y su sentimiento de identidad, como respuesta a la enriquecedora experiencia que supone enfrentarse a lo diferente en los ámbitos de la lengua y de la cultura. (Instituto Cervantes, 2002:12)

En todo acto comunicativo, según se recoge en el capítulo 5 de este texto, entrarían en juego dos tipos de competencias: las competencias generales y las comunicativas. Esta distinción no significa que exista un conjunto de competencias diferenciadas que se superponen o yuxtaponen. En realidad existe una única competencia compleja de la que el hablante se sirve en sus interacciones comunicativas. En esa competencia comunicativa entrarían tanto las competencias generales como las lingüísticas. Las competencias generales, que son las que remiten de manera más específica a la dimensión intercultural, se dividen, a su vez, en cuatro subcompetencias: conocimiento declarativo o saber, destrezas y habilidades (saber hacer), competencia existencial (saber ser) y capacidad de aprender (saber aprender). Dentro del conocimiento declarativo se encontrarían el conocimiento del mundo o cultura general, el conocimiento sociocultural y la consciencia intercultural. Pasaremos a exponer con detalle qué comprenden estos tres aspectos.

El conocimiento del mundo (ya sea derivado de la experiencia, de la educación o de las distintas fuentes de información) incluye: a) lugares, instituciones y organizaciones, personas, acontecimientos, objetos e intervenciones en distintos ámbitos. Es de especial importancia para el alumnado el conocimiento factual del país o países donde se habla el idioma objeto de aprendizaje, como pueden ser sus características geográficas, medioambientales, demográficas, económicas y políticas; y b) clases de entidades (concretas, abstractas, animadas e inanimadas, etc.) y sus propiedades y relaciones.

Por su parte, el conocimiento sociocultural, esto es, el conocimiento de la sociedad y cultura del país o países donde se habla la lengua constituye, en realidad, un aspecto del conocimiento del mundo, pero merece atención especial porque posiblemente no forme parte de la experiencia previa del alumnado y esté deformado por estereotipos. Entrarían en este subapartado, por consiguiente, las características distintivas de una sociedad concreta y de su cultura, incluyendo, por ejemplo, los siguientes temas: 1) La vida diaria: comida y bebida, horarios, modales; días festivos; el mundo del trabajo; y las actividades de ocio. 2) Las condiciones de vida: niveles de vida (incluyendo la diversidad regional, social y cultural); la vivienda; y la asistencia social. 3) Las relaciones personales: estructuras y relaciones familiares; relaciones entre sexos y entre generaciones; relaciones de raza y comunidad; relaciones entre grupos políticos y religiosos; estructura social y relación entre sus miembros. 4) Valores, creencias y actitudes respecto a clase social: riqueza, culturas regionales, instituciones, historia (personajes y acontecimientos representativos), minorías étnicas y religiosas, 
política, artes (música, literatura, teatro, canciones populares, etc.), religión. 5) El lenguaje corporal: conocimiento de las convenciones que rigen este aspecto, que forma parte de la competencia sociocultural del alumnado. 6) Convenciones sociales: puntualidad, regalos, ropa, aperitivos, bebidas y comidas, convenciones y tabúes referidos al comportamiento y a las conversaciones, despedida. Y, finalmente, 7) El comportamiento ritual en ceremonias y prácticas religiosas; nacimiento, matrimonio y muerte; comportamiento del público en representaciones y ceremonias; celebraciones, festividades, bailes, etc.

Llegamos así a la consciencia intercultural, elemento éste de primordial importancia en el enfoque intercultural y que remite de manera muy directa a las actitudes del alumnado con una connotación muy político-ideológica. En inglés se añade a esta expresión el adjetivo crítica (critical culture awareness). En francés se utiliza la expresión savoir s'engager, que es saber comprometerse, implicarse. En este componente del conocimiento declarativo figura la faceta de conexión entre culturas y análisis de la propia cultura y la extranjera. Esta consciencia intercultural supone, según el Marco europeo, una toma de conciencia del modo en que aparece la comunidad o comunidades desde la perspectiva de los demás, plagada frecuentemente de estereotipos (visión ésta contra la que se pretende luchar). Así, el conocimiento, la percepción y la comprensión de la relación entre el mundo propio y el de la comunidad cuya lengua se estudia (similitudes y diferencias) producen una consciencia intercultural que abarca la conciencia de la diversidad regional y social en los dos mundos.

Por lo que respecta a las destrezas y habilidades (saber hacer), podemos distinguir dos tipos: 1) Las destrezas y habilidades prácticas (destrezas sociales, de la vida, profesionales, de ocio); y las que a nuestro juicio tienen mayor importancia, 2) Las destrezas y habilidades interculturales (también llamadas aptitudes y saber hacer interculturales. Se incluyen aquí: a) la capacidad de establecer relaciones entre la propia cultura y la extranjera; b) la sensibilidad cultural y la capacidad de emplear una variedad de estrategias para establecer contacto con personas de otras culturas; c) la capacidad de ejercer el papel de mediador cultural entre la propia cultura y la extranjera y de abordar de modo eficaz los malentendidos y las situaciones conflictivas; y d) la capacidad de superar relaciones estereotipadas.

La competencia "existencial" (savoir-être) remite también al mundo de las actitudes y de los valores. Se incluyen aquí diversos factores: a) las actitudes, por ejemplo, la apertura hacia otras personas, ideas, pueblos, sociedades y culturas; la voluntad de relativizar la propia perspectiva cultural y el propio sistema de valores culturales; y la voluntad y capacidad de distanciarse de las actitudes convencionales en cuanto a la diferencia cultural; b) las motivaciones; c) los valores (éticos y morales); d) las creencias (religiosas, ideológicas, filosóficas); e) los estilos cognitivos; y, por último, f) los factores de personalidad.

En lo que se refiere a la capacidad de aprender (savoir-apprendre), puede afirmarse que en sentido general alude a la capacidad de observar y participar en nuevas experiencias y de incorporar conocimientos nuevos a los previos, cambiando estos últimos si es necesario. La capacidad para aprender lenguas se desarrolla en el curso de aprendizaje de las mismas. Dentro de esta capacidad, entrarían también varios componentes: 1) la reflexión sobre el sistema de la lengua y la comunicación; 2) la reflexión sobre el sistema fonético y las destrezas correspondientes; 3) las destrezas de estudio y, finalmente, 4) las destrezas heurísticas (de descubrimiento y análisis). Ejemplos de destrezas heurísticas serían: a) la capacidad del alumnado para adaptarse a la nueva experiencia; b) la capacidad del alumno para encontrar, comprender o transmitir nueva información; y c) la capacidad para usar las nuevas tecnologías. 
Estas competencias del Marco se corresponden con planteamientos muy análogos de especialistas en didáctica de las lenguas que han servido como fuente para la elaboración del documento analizado. Así, por ejemplo, en Byram (1997), -retomado, entre otros, por Sercu (2005) y Lund (2008) -, se establece un marco teórico para la competencia intercultural que consta de cinco saberes relacionados entre sí: el conocimiento (savoirs), las destrezas de interpretación y relación (savoir comprendre), las destrezas de descubrimiento e interacción (savoir apprendre/faire), las actitudes (savoir être) y la consciencia intercultural crítica (critical cultural awareness, savoir s'engager).

Sercu (2005: 2-5) retoma el marco conceptual de Byram (1997), señalando que la competencia intercultural está ligada a la comunicativa. La competencia comunicativa, de acuerdo con el Marco europeo, se refiere a la habilidad de una persona para desenvolverse en una lengua extranjera de un modo adecuado desde el punto de vista lingüístico, sociolingüístico y pragmático. La competencia comunicativa intercultural se construye sobre la base de la competencia comunicativa y la amplía para incorporar la competencia intercultural. Esta autora presenta los distintos componentes de la competencia intercultural agrupados en tres epígrafes: 1) conocimientos, 2) destrezas/comportamiento y 3) actitudes/rasgos. El primer saber, en realidad saberes, constituye la dimensión de conocimiento del marco conceptual. Comprende el conocimiento de los grupos sociales en el propio país y en el del interlocutor; así como el conocimiento de los procesos de interacción en los niveles individual y social. Saber aprender y saber comprender constituyen conjuntamente la dimensión de destrezas del marco conceptual. Saber aprender remite a la capacidad de aprender culturas y asignar significados a los fenómenos culturales de modo independiente (Byram y Zárate, 1997, cit. en Sercu, 2005). Ambos saberes se relacionan con la capacidad de interpretar y establecer relaciones entre las culturas. Saber hacer, por su parte, se refiere a la capacidad general de desenvolverse de un modo interculturalmente competente en situaciones de contacto intercultural, a tomar en cuenta la identidad cultural específica del interlocutor y desenvolverse de modo respetuoso y cooperativo. Saber ser y saber comprometerse se consideran conjuntamente, dado que se refieren a la disposición general que se caracteriza por un compromiso crítico con la cultura extranjera y la propia (saber comprometerse); y a la capacidad y disposición para abandonar actitudes y percepciones etnocéntricas, junto con la capacidad de establecer y mantener una relación entre la propia cultura y la extranjera (saber ser).

Guilherme (2000: 297) define la competencia intercultural como la "habilidad para interactuar de manera efectiva con gente de otras culturas que reconocemos como diferentes de la propia". Byram, Gribkova y Starkey (2002) insisten en que la adquisición de la competencia intercultural es un proceso que nunca se acaba ni puede ser totalmente perfecto por varias razones: en primer lugar, es imposible saber con anticipación cuál va a ser el conjunto de conocimientos necesarios en una interacción con personas pertenecientes a otras culturas; en segundo lugar, las identidades y valores sociales de una persona van cambiando a lo largo de toda la vida a medida que ésta se adhiere a distintos grupos sociales.

\section{El PAPEl del PRofesorado}

A la hora de desarrollar en el alumnado una competencia comunicativa intercultural, el profesorado debe tener claro cuáles son los objetivos que ha de perseguir. Hemos de tener 
presente que la dimensión intercultural apela más bien al mundo de las actitudes, destrezas y valores, aunque también incluye el conocimiento de la(s) cultura(s) extranjera(s). Al profesorado de lenguas extranjeras le compete el ejercicio de la mediación profesional entre culturas. Para ello debe tener presente que, entre sus objetivos, han de figurar los siguientes: 1) hacer adquirir al alumnado una competencia tanto lingüística como intercultural; 2) prepararlo para establecer relaciones con personas pertenecientes a otras culturas y aceptar a esas personas como individuos poseedores de puntos de vista, valores y comportamientos diferentes; 3) ayudarle a valorar el carácter enriquecedor de este tipo de experiencias y relaciones; 4) hacerle captar la relación entre su propia cultura y otras culturas; 5) suscitar en él el interés y la curiosidad hacia la alteridad; y 6) hacerle a tomar conciencia del modo en que otros lo perciben a él mismo y a su cultura (Byram, Gribkova y Starkey, 2002).

Es importante señalar que, para alcanzar los anteriores objetivos, se requiere utilizar una gama de recursos didácticos lo más variada posible: textos originales, grabaciones de audio y video, fotografías, gráficos, dibujos, folletos turísticos, canciones, películas, periódicos, revistas, etc. Hoy en día Internet proporciona una fuente inagotable de materiales, por lo que consideramos esencial la incorporación de las nuevas tecnologías. En esta tarea de búsqueda de información debe implicarse al propio alumnado.

En otro orden de cosas, el profesorado debe ser un profesional crítico y reflexivo, capaz de promover la tolerancia y el respeto hacia el "otro". Su papel no es el de mero transmisor de conocimientos de la cultura extranjera. Él mismo debe convertirse en un "aprendiz intercultural", capaz de promover el trabajo autónomo del alumnado y de adquirir las mismas destrezas y actitudes que pretende desarrollar en este último.

Por último, volviendo una vez más al currículum de lenguas extranjeras vigente en España para la etapa de Educación Secundaria Obligatoria, nos encontramos con que éste remite a diversas competencias básicas que el alumnado ha de adquirir: competencia en comunicación lingüística, competencia para aprender a aprender, competencia social y ciudadana, competencia en el tratamiento de la información y competencia digital, competencia cultural y artística y, finalmente, autonomía e iniciativa personal. Pese a que todas ellas se relacionan, a nuestro modo de ver, con la dimensión intercultural, nos gustaría detenernos en dos: la competencia social y ciudadana y la competencia artística y cultural. (Real Decreto 1631/2006: 742-743).

Respecto a la competencia social y ciudadana, se apunta que las lenguas a) son vehículo de comunicación y transmisión cultural; y b) favorecen el respeto, el interés y la comunicación con personas que hablan otras lenguas y el reconocimiento y la aceptación de las diferencias culturales. Además, desde un punto de vista metodológico, dado que el trabajo en grupo y en parejas es importante en esta materia, se aprende así a participar, a expresar las ideas propias y a escuchar las de los demás. Se desarrolla pues la capacidad de diálogo, de negociación de significados y de llegar a acuerdos. De este modo se favorece "aprender de y con los demás" (Ibidem: 743).

Finalmente se destaca que la materia incluye un acercamiento a manifestaciones culturales de la lengua y países en que se habla, contribuyendo así a la adquisición de la competencia artística y cultural. Por otra parte, si se facilita la expresión de opiniones, gustos ante manifestaciones culturales y se promueve la realización y representación de simulaciones y narraciones, se contribuye a la apreciación de la diversidad cultural. 
Como puede verse, estas dos competencias destacan aspectos metodológicos propios de la enseñanza de lenguas que apuntan claramente a la formación de ciudadanas y ciudadanos democráticos, respetuosos con la diversidad, tolerantes y conscientes de la propia identidad cultural y de la de los demás.

\section{Bibliografía}

Abdallah-Pretceille, M. (1998). "Apprendre une langue, apprendre une culture, apprendre l'altérité", en Les Cahiers Pédagogiques, 360: 49-51.

Areizaga, E. (2001).”Cultura para la formación de la competencia comunicativa intercultural: el enfoque formativo", en Revista de Psicodidáctica, 11-12: 157-170.

Buttjes, D. y Byram, M. (eds.) (1991). Mediating Languages and Cultures. Clevedon: Multilingual Matters.

Byram, M. (1997). Teaching and Assessing Intercultural Communicative Competence. Clevedon: Multilingual Matters.

Byram, M. (coord.) (2003). La compétence interculturelle. Strasbourg: Conseil de l'Europe.

Byram, M. y Fleming, M. (2001). Perspectivas interculturales en el aprendizaje de idiomas. Enfoques a través del teatro y la etnografía. Madrid: Cambridge University Press.

Byram, M.; Gribkova, B. y Starkey, H. (2002). Développer la dimension interculturelle dans l'enseignement des langues. Une introduction à l'usage des enseignants. Strasbourg: Conseil de l'Europe.

Byram, M.; Morgan, C. y otros (1994). Teaching-and-Learning Language-and-Culture. Clevedon: Multilingual Matters.

Byram, M. y Risager, K. (1999). Language Teachers, Politics and Cultures. Clevedon: Multilingual Matters.

Byram, M.; Nichols, A. y Stevens, D. (2001). Developping Intercultural Competence in Practice. Clevedon: Multilingual Matters.

Byram, M. y Zarate, G. (1997). "Définitions, objectifs et évaluation de la compétence socioculturelle" en M. Byram; G. Zarate y G. Neuner. La compétence socioculturelle dans l'apprentissage et l'enseignement des langues. Strasbourg: Conseil de 1'Europe, 7-36.

Eurydice (2001). L'enseignement des langues étrangères en milieu scolaire en Europe. Bruselas: Les Éditions européennes.

Guilherme, M. (2000). "Intercultural competence" en M.Byram (ed.). Routledge Encyclopedia of Language Teaching and Learning. London and New York: Routledge, 297-300.

Instituto Cervantes (2002). Marco de referencia europeo para el aprendizaje, la enseñanza y la evaluación de lenguas. Madrid: Instituto Cervantes.

Isisag, K.U. (2010). "The Acceptance and Recognition of Cultural Diversity in Foreign Language Teaching", in Academik Bakis, Cilt 4. Sayi 7: 251-260.

Kramsch, C. (1993). Context and Culture in Language Teaching. Oxford: Oxford University Press.

Kramsch, C. (1998). Language and Culture. Oxford: Oxford University Press.

Kramsch, C. (2001). "El privilegio del hablante intercultural" en M. Byram y M. Fleming. Perspectivas interculturales en el aprendizaje de idiomas. Enfoques a través del teatro y la etnografía. Madrid: Cambridge University Press, 27-37.

Lund, R.E. (2008). "Intercultural competence -an aim for the teaching of English in Norway?". Acta Didactica Norge, vol. 2, 1, art. 9: 1-16. 
Neuner, G. (2003). "Les mondes socioculturels intermédiaires dans l'enseignement et l'apprentissage des langues vivantes", en M. Byram (coord.). La competence interculturelle, 15-66.

Paricio, M. S. (2004). "Dimensión intercultural en la enseñanza de las lenguas y formación del profesorado", en Revista Iberoamericana de Educación, 34 (4): 1-12.

Paricio, M. S. (2011). "Contribución de las lenguas extranjeras al desarrollo de actitudes de tolerancia y respeto hacia el 'otro"', en Linguarum Arena, 2: 79-89.

Real Decreto 1631/2006, de 29 de diciembre, por el que se establecen las enseñanzas mínimas correspondientes a la Educación Secundaria Obligatoria. BOE de 5 de enero de 2007.

Rico, A. M. (2005). "De la competencia intercultural en la adquisición de una segunda lengua o lengua extranjera: conceptos, metodología y revisión de métodos". Porta Linguarum 3: 79-94.

Risager, K. (2001). "La enseñanza de idiomas y el proceso de integración europea", en M. Byram y M. Fleming. Perspectivas interculturales en el aprendizaje de idiomas. Madrid: Cambridge University Press, 242-253.

Sercu, L. (2002). "Autonomous Learning and the Acquisition of Intercultural Communicative Competence: Some Implications for Course Development", in Language, Culture and Curriculum 15, 1: 61-74.

Sercu, L. (2004). "Intercultural Communicative Competence in Foreign Language Education", en K. van Esch y O. St. John (eds.). New Insights into Foreign Language Learning and Teaching, Frankfurt am Main: Peter Lang, 115-130.

Sercu, L. (2005). "Teaching Foreign Languages in an Intercultural World", en L. Sercu, E. Bandura, P. Castro, L. Davcheva, Ch. Laskaridou, U. Lundgren, Ma del C. Méndez García y P. Ryan. Foreign Language Teachers and Intercultural Competence. An International Investigation. Clevedon: Multilingual Matters, 1-18.

Starkey, H. (2003). "Compétence interculturelle et education à la citoyenneté démocratique: incidences sur la méthodologie d'enseignement des langues", en M. Byram (coord.). La competence interculturelle, 67-88.

Starkey, H. (2005). Language teaching for democratic citizenship, en A. Osler y H. Starkey (coords.). Citizenship and Language Learning: international perspectives, 23-39.

Zárate, G. (1986). Enseigner une culture étrangère. Paris: Hachette.

Zárate, G. (1995). Représentations de l'étranger et didactique des langues. Paris: Didier. 